\title{
MODULN, DIE IN JEDER ERWEITERUNG EIN KOMPLEMENT HABEN
}

\author{
HELMUT ZÖSCHINGER
}

\section{Einleitung.}

Ist ein Modul $M$ injektiv oder artinsch, so hat er in jeder Erweiterung $M \subset N$ ein (additives) Komplement, d.h. ein minimales Element in der Menge

$$
\{T \mid T \subset N \text { und } T+M=N\} \text {. }
$$

Für artinsches $M$ gilt sogar stärker: Ist $X+M=N$, so gibt es ein Komplement von $M$ in $N$, das in $X$ enthalten ist, d.h. $M$ hat genügend viele Komplemente in $N$. In der vorliegenden Arbeit sollen für einen Modul $M$ diese "äußeren« Komplementiertheits-Eigenschaften untersucht werden:

(E) $M$ hat in jeder Erweiterung ein Komplement. (EE) $M$ hat in jeder Erweiterung genügend viele Komplemente.

Für spezielle Modulkategorien gelingt es, diese beiden äußeren Eigenschaften durch "innere» zu charakterisieren, und unser Hauptergebnis lautet für $R=\mathrm{Z}$ : Eine abelsche Gruppe $M$ hat genau dann die Eigenschaft (E), wenn sie von der Form ist $M \cong D \times \Pi_{p} A_{p}$, wobei $D$ teilbar ist und $A_{p}$, für jede Primzahl $p$, eine beschränkte $p$-Gruppe; $M$ hat genau dann die Eigenschaft (EE), wenn der divisible Anteil von $M$ artinsch ist, und der reduzierte Anteil von $M$ beschränkt.

In Abschnitt 1 werden einige elementare Tatsachen über die Eigenschaften (E) und (EE) zusammengestellt, insbesondere ergibt sich zwischen beiden folgender einfache Zusammenhang: Ein Modul $M$ hat genau dann die Eigenschaft (EE), wenn jeder Untermodul von $M$ die Eigenschaft (E) hat. Hinreichend für die Eigenschaft (E) ist, daß $R / \operatorname{Ann}_{R}(M)$ ein perfekter Ring ist. Als Anwendung zeigen wir in (1.6), daß über einem kommutativen noetherschen Ring $R$ ein halbeinfacher Modul $M_{R}$ genau dann die Eigenschaft (E) hat, wenn fast alle seine Isotypiekomponenten Null sind, und das ist äquivalent damit, daß $M_{R}$ algebraisch kompakt ist.

Jeder linear-kompakte Modul hat die Eigenschaft (EE). In Abschnitt 2 wird für Spezialfälle die Umkehrung untersucht: Ist $R$ ein noetherscher

Eingegangen am 20. Oktober 1974. 
Integritätsring, und hat der Quotientenkörper $K$ als $R$-Modul die Eigenschaft (EE), so ist er bereits linear-kompakt, und das ist äquivalent damit, daß $K_{R}$ selbstprojektiv ist. Ist $R$ sogar dedekindsch, so lassen sich die $R$-Moduln mit der Eigenschaft (EE) vollständig angeben: Sie sind die direkte Summe aus einem linear-kompakten und einem beschränkten Modul.

Ziel von Abschnitt 3 ist die Bestimmung aller Moduln mit der Eigenschaft (E) über einem diskreten Bewertungsring $R$ : Sie sind algebraisch kompakt, und nach Theorem (3.5) hat ein $R$-Modul $M$ genau dann die Eigenschaft (E), wenn er von der Form ist $\left(R^{*}\right)^{a} \times D \times B$, wobei $R^{*}$ die Vervollständigung von $R$ ist, $a \geqq 0, D$ teilbar und $B$ beschränkt. Ist $R$ sogar vollständig, so hat man folgende Charakterisierung: $M$ hat genau dann die Eigenschaft (E), wenn es in seiner injektiven Hülle ein Komplement hat, und das ist äquivalent damit, daß $\mathrm{Ra}(M)$ ein Komplement in $M$ hat. Für diese letztere, "innere "Eigenschaft wird in (3.1), auch über unvollständigem $R$, noch eine Reihe dazu äquivalenter Aussagen angegeben.

Zur Frage, wann aus der Existenz eines Komplementes für das Radikal bereits die Kleinheit des Radikals folgt, erhalten wir in Abschnitt 4 über einem beliebigen Ring $R$ : Ist $M$ ein $\Sigma$-selbstprojektiver Modul, ist $U \subset \operatorname{Ra}(M)$, und hat $U$ ein Komplement in $M$, so ist $U$ bereits klein in $M$. Dies ist eine Verallgemeinerung der wohlbekannten Tatsachen, daß in einem projektiven komplementierten Modul das Radikal klein ist, und daß ein projektiver radikalvoller Modul bereits Null ist.

Ziel von Abschnitt 5 ist die Bestimmung aller Moduln mit der Eigenschaft (E) über einem nicht-lokalen Dedekindring $R$ : Sie sind wieder algebraisch kompakt, aber im Unterschied zum lokalen Fall in Abschnitt 3 ist jetzt jeder torsionsfreie $R$-Modul mit der Eigenschaft (E) bereits teilbar, denn in (5.5) zeigen wir: $M$ hat genau dann in jeder Erweiterung $N$, mit $N / M$ torsionsvoll, ein Komplement, wenn $M / T(M)$ teilbar ist und wenn in $T(M)$ das Radikal ein Komplement hat. Diese Eigenschaft, zusammen mit $\operatorname{Ext}_{R}{ }^{1}(K, M)=0$ ( $K$ der Quotientenkörper), ist sogar äquivalent mit (E), und daraus folgt unser Theorem (5.6), das vollkommen dem am Anfang zitierten Struktursatz über $R=Z$ entspricht.

\section{Allgemeines.}

Alle Ringe sind assoziativ mit Eins, und die Moduln unitäre Rechtsmoduln. $M_{R}$ heißt nach Kasch-Mares [7] komplementiert, wenn jeder Untermodul ein Komplement in $M$ hat. $M_{R}$ heißt nach Golan [5] supplementiert, wenn jeder Untermodul genügend viele Komplemente in $M$ hat 
(siehe zu diesen Begriffen auch [15], [16]). $M_{R}$ heißt linear-kompakt (in der diskreten Topologie), wenn jedes endlich lösbare System $\left(m_{i}, U_{i}\right)_{i \in I}$ von Kongruenzen schon global lösbar ist, d.h. ein $m_{0} \in M$ hat mit $m_{i}-m_{0} \in U_{i}$ für alle $i$. Bei Onodera [12, Theorem 5] und Sandomierski [13, Proposition 2.6] wird gezeigt, daß jeder linear-kompakte Modul komplementiert ist.

Der Beweis von Sandomierski läßt sich so lesen: Ist $M$ linear-kompakt, $M \subset N$ und $\left(T_{i} \mid i \in I\right)$ eine nach unten gefilterte Familie von Untermoduln von $N$, so gilt

$$
\left(\bigcap T_{i}\right)+M=\bigcap\left(T_{i}+M\right) .
$$

Also ist insbesondere für jedes $X+M=N$ die Menge

$$
\{T \mid T \subset X \text { und } T+M=N\}
$$

induktiv nach unten geordnet und hat nach Zorn ein minimales Element. Ergebnis: Jeder linear-kompakte Modul hat die Eigenschaft (EE).

Lemma 1.1. Für einen linear-kompakten Modul M gilt:

(a) $M$ ist genau dann noethersch, wenn jeder radikalvolle Untermodul von $M$ Null ist.

(b) $M$ ist genau dann artinsch, wenn jeder sockelfreie Faktormodul von $M$ Null ist.

Beweis. Unter dem Radikal eines Moduls $X$ verstehen wir $\operatorname{Ra}(X)=$ der Durchschnitt aller maximalen Untermoduln = die Summe aller kleinen Untermoduln. Entsprechend ist $\mathrm{So}(X)=$ der Durchschnitt aller großen Untermoduln $=$ die Summe aller einfachen Untermoduln. $X$ heißt radikalvoll bzw. sockelfrei wenn $\operatorname{Ra}(X)=X$ bzw. So $(X)=0$.

Damit ist klar, daß aus noethersch (artinsch) die Bedingung für die Untermoduln (Faktormoduln) folgt. - Hat bei (a) umgekehrt $M$ keine radikalvollen Untermoduln, so ist $\mathrm{Ra}(M)$ klein in $M$, denn ist $X$ ein Komplement von $\mathrm{Ra}(M)$ in $M$, und $Y$ ein Komplement von $X$ in $M$, so ist mit $M / X$ auch $Y$ radikalvoll, also $Y=0$ und $X=M$. Weil weiter $M / \operatorname{Ra}(M)$ halbeinfach und linearkompakt ist, ist es endlich erzeugt, also auch $M$. Das gleiche Argument zeigt, daß jeder Untermodul von $M$ endlich erzeugt, also $M$ noethersch ist. - Ebenso zeigt man bei (b), daß jeder Faktor von $M$ endlich koerzeugt ist (siehe [12, Proposition 6]).

Bemerkung. Die gleichen Aussagen gelten, wenn man die Voraussetzung $" M$ linear-kompakt» ersetzt durch $" \mathscr{L}(M)$ totalgeordnet». 
Folaterung. Über einem noetherschen Integritätsring mit Krull-Dimension 1 ist ein Modul genau dann artinsch, wenn er linear-kompakt und torsionsvoll ist.

Lemma 1.2. Ein Modul $M$ hat die Eigenschaft (EE) genau dann, wenn jeder Untermodul von $M$ die Eigenschaft (E) hat.

Bewers. Hat jeder Untermodul von $M$ die Eigenschaft (E), und ist $X+M=N$, so hat insbesondere $X \cap M$ ein Komplement $V$ in $X$, und dann ist $V$ auch ein Komplement von $M$ in $N$. - Ist umgekehrt $M$ ein Modul mit der Eigenschaft (EE), $U$ ein Untermodul von $M$ und $U \subset N$, so bilde man die Fasersumme

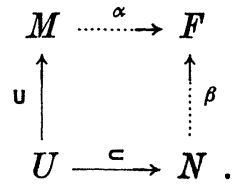

Mit $M^{\prime}=\mathrm{Bi} \alpha$ und $N^{\prime}=\mathrm{Bi} \beta$ folgt $F=M^{\prime}+N^{\prime}$, so daß nach Voraussetzung $M^{\prime}$ ein Komplement $V$ in $F$ hat mit $V \subset N^{\prime}$. Nun ist $V$ auch ein Komplement von $M^{\prime} \cap N^{\prime}$ in $N^{\prime}$, und damit $\beta^{-1}(V)$ ein Komplement von $U$ in $N$.

Folanduna. Ein Modul mit der Eigenschaft (EE) ist supplementiert.

Lemma 1.3.

(a) Die Eigenschaft (E) vererbt sich auf direkte Summanden.

(b) Sei $A \subset B \subset C$ mit $C / A$ injektiv. Hat dann $B$ die Eigenschaft (E), so hat sie auch $B / A$.

(c) Sei $A \subset B$. Haben dann $A$ und $B / A$ die Eigenschaft (E), so hat sie auch $B$.

Bewers. (a) Besitze $A \times M$ die Eigenschaft (E) und sei $M \subset N$. Dann hat $A \times M$ ein Komplement $V$ in $A \times N$, und weil der Kern des Epimorphismus $g: A \times N \rightarrow N$ enthalten ist in $A \times M$, folgt nach [15, Lemma 1.2], daß $g(V)$ ein Komplement von $M$ in $N$ ist. (b) Ist $B / A \subset N$, so gibt es, weil $C / A$ injektiv, das folgende kommutative Diagramm mit exakten Zeilen:

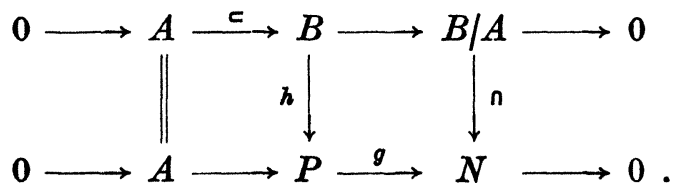


Weil nun $B$ die Eigenschaft (E) hat und $h$ ein Monomorphismus ist, hat Bi h ein Komplement $V$ in $P$, und wie bei (a) folgt, daß $g(V)$ ein Komplement von $B / A$ in $N$ ist. (c) $\mathrm{Zu} B \subset N$ hat man nach Voraussetzung ein Komplement $V / A$ von $B / A$ in $N / A$, und ein Komplement $W$ von $A$ in $V$. Aus dem wesentlichen Epimorphismus

$$
W \rightarrow V \mid A \rightarrow N / B
$$

folgt, daß $W$ auch ein Komplement von $B$ in $N$ ist.

Beispiel zu (b). Sei $R$ ein unendliches Produkt von Körpern. Dann sind die $R$-Moduln mit der Eigenschaft (E) genau die injektiven, denn alle $R$-Moduln sind radikalfrei. Damit hat $R_{R}$ die Eigenschaft (E), nicht aber jeder Faktormodul von $R_{R}$.

Lemma 1.4. Sei $I$ ein zweiseitiges Ideal in $R$ und $\bar{R}=R / I$. Dann gilt:

(a) Hat $M \in \operatorname{Mod}-R$ die Eigenschaft (E), und ist $M I=0$, so hat auch $M_{\bar{R}} \in \operatorname{Mod}-\bar{R}$ die Eigenschaft (E).

(b) Ist der Ring $\bar{R}$ rechts-perfekt, und ist $M \in \operatorname{Mod}-R$ mit $M I=0$, so hat $M$ die Eigenschaft (E).

Beweis. (a) Aus $M_{\bar{R}} \subset N$ in Mod $-\bar{R}$ folgt $M \subset N_{R}$, so daß nach Voraussetzung $M$ ein Komplement $V$ in $N_{R}$ hat. Es folgt, daß $V_{\bar{R}}$ ein Komplement von $M_{\bar{R}}$ in $N$ ist. (b) Sei $\bar{R}$ rechts-perfekt, d.h. jeder Rechts- $\bar{R}$ Modul komplementiert, und $M$ wie angegeben, $M \subset N$ in Mod $-R$. Weil $N / N I$ komplementiert ist, gibt es ein $N I \subset V \subset N$, so daß $V / N I \mathrm{Kom}$ plement von $(M+N I) / N I$ in $N / N I$ ist. Nun ist $V$ auch ein Komplement von $M$ in $N$, denn die Summe ist klar, und aus $T+M=N$ mit $T \subset V$ folgt $N I \subset T$ (wegen $M I=0$ ), also $T=V$.

Folgerung. Über einem noetherschen Integritätsring mit Krull-Dimension 1 hat jeder beschränkte Modul die Eigenschaft (E).

Wir wollen noch, in diesem allgemeinen Teil, die Eigenschaft (E) für den $R$-Modul $R^{(\mathrm{N})}$ und für halbeinfache $R$-Moduln untersuchen. Dazu wird folgender Begriff benötigt: Ein Modul $M$ heißt reduziert, wenn jeder radikalvolle Untermodul von $M$ Null ist. - Die Klasse der reduzierten $R$-Moduln ist gegenüber Untermoduln, Gruppenerweiterungen und beliebigen direkten Produkten abgeschlossen. Für Faktormoduln erhält man : Ist $M$ reduziert, und $U \subset M$ derart, daß $U$ in jedem Zwischenmodul zu $M$ ein Komplement hat, so ist auch $M / U$ reduziert. 
SaTz 1.5. Der Ring $R$ ist genau dann rechts-perfekt, wenn $R_{R}$ reduziert ist und der R-Rechts-Modul $R^{(\mathrm{N})}$ die Eigenschaft (E) hat.

BEwErs. Es ist wohlbekannt, daß über einem rechts-perfekten Ring $R$ jeder $R$-Rechts-Modul reduziert und komplementiert ist. Zur Umkehrung zeigen wir:

(1) Ist $M$ ein projektiver, reduzierter Modul mit der Eigenschaft (E), so hat er ein kleines Radikal: $\mathrm{Zu} X+\operatorname{Ra}(M)=M$ gibt es nämlich ein $\alpha \in \operatorname{End}(M)$ mit

$$
\operatorname{Bi} \alpha \subset X \text { und } \operatorname{Bi}(1-\alpha) \subset \operatorname{Ra}(M) .
$$

Es folgt, daß $\alpha$ ein Monomorphismus ist [2, Theorem 3, oder auch der 2. Schritt im Beweis von 4.1], so daß Bi $\alpha$ ein Komplement $Y$ in $M$ hat. Als wesentliche Überdeckung von $M / \mathrm{Bi} \alpha$ ist auch $Y$ radikalvoll, also $Y=0$ und $X=M$.

(2) Sei nun $R_{R}$ reduziert (also auch jeder projektive $R$-Rechts-Modul), und besitze $F=R^{(\mathrm{N})}$ die Eigenschaft $(\mathrm{E})$. Ist $\left(x_{i} \mid i \in \mathrm{N}\right)$ die kanonische Basis von $F$, so genügt es zur Rechts-Perfektheit von $R$ zu zeigen (nach [1, Lemma 1.3]), daß für jede Folge $\left(a_{i} \mid i \in \mathrm{N}\right)$ von Elementen aus $R$ der Untermodul

$$
M=\sum_{i=1}^{\infty}\left(x_{i}-x_{i+1} a_{i}\right) R
$$

in $F$ direkter Summand ist: Weil $M \cong F$ die Eigenschaft (E) hat, ist $\mathrm{Ra}(M)$ nach (1) klein in $M$, und $M$ hat ein Komplement $V$ in $F$. Aus $\operatorname{Ra}(M)=M \cap \operatorname{Ra}\left(F^{\prime}\right)$ folgt $V \cap M \subset \operatorname{Ra}(M)$, also sogar $V \cap M$ klein in $M$, so daß endlich $V$ und $M$ gegenseitig Komplemente in $F$ sind, und damit ist $F=V \oplus M$ (siehe etwa [16, Lemma 1.3]).

SATz 1.6. Ist $R$ kommutativ und noethersch, so sind für den halbeinfachen $R$-Modul $M$ äquivalent:

(i) $M$ hat die Eigenschaft (E).

(ii) $M$ ist algebraisch kompakt.

(iii) Fast alle Isotypiekomponenten von $M$ sind $N$ ull.

BeweIs. (i) $\Rightarrow$ (iii): Ist $M=\amalg_{i \in I} M_{i}$ die Zerlegung von $M$ in seine Isotypiekomponenten, und definiert man dazu $N=\Pi_{i \in I} M_{i}$, so gilt für jedes maximale Ideal $\mathfrak{m} \subset R$, weil es endlich erzeugt ist,

$$
N \mathfrak{m}=\Pi_{i \in I}\left(M_{i} \mathfrak{m}\right),
$$

also $N \mathfrak{m}+M=N$. Damit ist $N / M$ radikalvoll, und ist nun $V$ ein Komplement von $M$ in $N$, so ist auch $V$ radikalvoll, also Null (weil $N$ reduziert), und aus $M=N$ folgt $M_{i}=0$ für fast alle $i \in I$. 
(iii) $\Rightarrow$ (ii): Wir zeigen, daß $M$ reininjektiv ist, d.h. jede reine Erweiterung $M \subset N$ zerfällt: Nach $(1.4, \mathrm{~b})$ hat $M$ die Eigenschaft (E), also gibt es ein Komplement $V$ von $M$ in $N$. Aus $V \cap M$ klein in $N$ folgt

$$
V \cap M \subset \operatorname{Ra}(N) \cap M=\operatorname{Ra}(M)=0,
$$

also $V \oplus M=N$.

(ii) $\Rightarrow$ (i): Ist $M \subset N$, so ist

$$
X=(M+\operatorname{Ra}(N)) / \operatorname{Ra}(N)
$$

wieder halbeinfach und algebraisch kompakt. Nun sind in jedem radikalfreien Modul die halbeinfachen Untermoduln rein, so daß hier $X$ als reiner Untermodul von $N / \operatorname{Ra}(N)$ sogar direkter Summand ist. Aus

$$
V / \operatorname{Ra}(N) \oplus X=N / \operatorname{Ra}(N)
$$

folgt wie in $(1.4, \mathrm{~b})$, daß $V$ ein Komplement von $M$ in $N$ ist, denn zu $T+M=N$ ist $N / T \cong M / T \cap M$ radikalfrei, also $\operatorname{Ra}(N) \subset T$.

\section{Linear-Kompaktheit und die Eigenschaft (EE).}

Das Ergebnis (1.6), ebenso jeder perfekte Ring, zeigt, daß ein Modul mit der Eigenschaft (EE) nicht linear-kompakt zu sein braucht. Es soll in diesem Abschnitt die Umkehrung untersucht werden für zwei Spezialfälle: Für den Quotientenkörper eines noetherschen Integritätsringes, und für alle Moduln über einem Dedekindring.

Lemma 2.1. Sei $R$ ein Integritätsring, mit Quotientenkörper $K$, und sei in $M_{R}$ jeder teilbare torsionsvolle Untermodul Null. Dann gilt:

Hat $M_{R}$ die Eigenschaft (E), so ist $\operatorname{Ext}_{R}{ }^{1}(K, M)=0$.

BeweIs. (1) Ist $\beta: H \rightarrow K$ ein wesentlicher Epimorphismus, so ist $\mathrm{Ke} \beta$ gleich dem Torsionsuntermodul $T(H)$. Mit $K$ ist nämlich auch $H$ teilbar, und weil $K$ torsionsfrei, ist auch $\operatorname{Ke} \beta$ teilbar. Damit ist $\operatorname{Ke} \beta / T(H)$ ein injektiver, kleiner Untermodul von $H / T(H)$, also Null.

(2) Sei nun $M$ wie angegeben, und $M \subset N$ mit $N / M \cong K$. Nach Voraussetzung hat $M$ ein Komplement $V$ in $N$, und man zeigt, daß $V$ sogar direktes Komplement ist: Der wesentliche Epimorphismus $V \rightarrow N / M$ hat den Kern $V \cap M$, der nach (1) teilbar und torsionsvoll ist, also Null.

BEMERKung. Ist jede wesentliche Überdeckung des Quotientenkörpers schon ein Isomorphismus, so ist $\operatorname{Ext}_{R}{ }^{1}(K, M)=0$ für jeden $R$-Modul $M$ 
mit der Eigenschaft (E). Zum Beispiel trifft das für jeden Dedekindring $\mathrm{zu}$, allgemeiner für jeden noetherschen Integritätsring $R$ mit $\operatorname{dim}(R)=1$, denn nach [9, Theorem 3.3] ist dann in jedem teilbaren $R$-Modul der Torsionsuntermodul direkter Summand, also unser $\beta$ in (1) ein Isomorphismus.

SATz 2.2. Für einen Integritätsring $R$ mit Quotientenkörper $K \neq R$ sind äquivalent:

(a) $K$ ist als $R$-Modul selbstprojektiv.

(b) $K \times K$ ist als $R$-Modul supplementiert.

(c) Ist $M \subset K \times K$, und hat $M$ keine teilbaren Untermoduln, so ist $M$ bereits klein in $K \times K$.

(d) $\operatorname{Ext}_{R}{ }^{1}(K, U)=0$ für alle $U \subset K$.

Falls $R$ noethersch, ist das weiter äquivalent mit:

(i) $K_{R}$ hat die Eigenschaft (EE).

(ii) $R_{R}$ hat die Eigenschaft (E), und $\operatorname{dim}(R)=1$.

(iii) $R$ ist lokal und vollständig, und $\operatorname{dim}(R)=1$.

(iv) $K_{R}$ ist linear-kompakt.

BEweIs. Ein $R$-Modul $X$ heißt bekanntlich selbstprojektiv, wenn für jeden Untermodul $U$ von $X$ die Abbildung

$$
\operatorname{Hom}_{R}(X, X) \rightarrow \operatorname{Hom}_{R}(X, X / U)
$$

surjektiv ist. Es ist dann auch $X^{2}=X \times X$ selbstprojektiv, und zu $V+U=X$ gibt es stets einen Endomorphismus $\alpha$ von $X$ mit $\operatorname{Bi} \alpha \subset V$ und $B i(1-\alpha) \subset U$ (siehe dazu [4] oder auch [16]).

(a) $\Rightarrow$ (b): Weil $K$ selbstprojektiv, ist jeder echte Untermodul von $K$ klein in $K$, also ist $K$ komplementiert. Damit ist aber auch $K^{2}$ selbstprojektiv und komplementiert, also nach [16, Lemma 1.3] supplementiert.

(b) $\Rightarrow$ (c): Sei $M$ wie angegeben, $V$ ein Komplement von $M$ in $K^{2}$, und $W$ ein Komplement von $V$ in $K^{2}$ mit $W \subset M$. Dann ist $W$ teilbar, also $W=0$, und aus $V=K^{2}$ folgt $M$ klein in $K^{2}$.

(c) $\Rightarrow$ (d): Sei $U \subsetneq K$, und weiter $U \subset M$ mit $M / U \cong K$. Dann ist $M$ torsionsfrei mit $\operatorname{Rang}(M) \leqq 2$, also o.B.d.A. sogar $M \subset K^{2}$. Weil $M$ einen von Null verschiedenen injektiven Faktor hat, ist $M$ nicht klein in $K^{2}$, so daß es nach Voraussetzung einen teilbaren Modul $M^{\prime}$ gibt mit $0 \neq M^{\prime} \subset M$. Wir behaupten, daß $M^{\prime}$ ein direktes Komplement von $U$ in $M$ ist: Weil $M^{\prime} \mid M^{\prime} \cap U$ torsionsfrei, ist auch $M^{\prime} \cap U$ teilbar, also Null; und weil $\left(M^{\prime}+U\right) / U$ ein von Null verschiedener teilbarer Untermodul von $M / U$ ist, gilt auch noch $M^{\prime}+U=M$. 
(d) $\Rightarrow$ (a): Für jedes $U \subset K$ ist exakt die Folge

$$
\operatorname{Hom}_{R}(K, K) \rightarrow \operatorname{Hom}_{R}(K, K / U) \rightarrow 0,
$$

und das bedeutet gerade die Selbstprojektivität von $K$.

Der zweite Teil des Satzes ist, weil $R$ noethersch, nur eine Umformulierung, in den Komplementiertheitsbegriff, von Ergebnissen von Matlis aus [10].

Insbesondere gilt (d) $\Leftrightarrow$ (iii) nach [10, Theorem 4].

(i) $\Rightarrow$ (ii): Nur die Aussage über die Krull-Dimension ist noch zu beweisen : Weil $K_{R}$ komplementiert, ist jeder echte Untermodul klein in $K$, also jeder echte Faktormodul von $K$ direkt unzerlegbar. Nach [10, Theorem 2] ist damit $\operatorname{dim}(R)=1$.

(ii) $\Rightarrow$ (iii): $R$ ist lokal, denn ist $x \in R$ nicht invertierbar und $V$ ein Komplement von $x R$ in $R_{R}$, so ist $V$ zyklisch und idempotent, also direkter Summand in $R_{R}$, also $V=R$, also $x \in \mathrm{Ra}(R) .-R$ ist vollständig, denn ist $V$ ein Komplement von $R_{R}$ in $R^{*}$, der Vervollständigung von $R$, so ist mit $R^{*} / R$ auch $V$ radikalvoll, also $V=0$ (weil $R^{*}$ reduziert), also $R=R^{*}$.

(iii) $\Rightarrow$ (iv): Nach [14, Theorem 3] ist $R_{R}$ linear-kompakt. Außerdem ist $K / R$ nach [10, Theorem 1] artinsch, also auch $K_{R}$ linear-kompakt. (iv) $\Rightarrow$ (i): klar.

Wegen $\operatorname{dim}(R)=1$ stimmen die radikalvollen $R$-Moduln mit den teilbaren $R$-Moduln überein, so daß man mit (1.1) erhält:

Folgerung [10, Corollary 3, p. 579]. Ist $R$ ein noetherscher Integritätsring wie im Satz, und ist $M$ ein torsionsfreier $R$-Modul von endlichem Rang, so ist $M$ von der Form $X \times K^{n}$ mit $X$ endlich erzeugt.

SAtz 2.3. Sei $R$ ein Dedekindring, kein Körper. Dann sind für $M_{R}$ äquivalent:

(i) $M$ hat die Eigenschaft (EE).

(ii) Jeder Untermodul von $M$ ist kotorsion.

(iii) $M$ ist direkte Summe aus einem linear-kompakten und einem beschränkten $R$-Modul.

Beweis. Ein $R$-Modul $X$ heißt bekanntlich kotorsion, wenn $\operatorname{Ext}_{R}{ }^{1}(A, X)=0$ für alle torsionsfreien $A_{R}$. Weil $R$ dedekindsch, ist das äquivalent mit $\operatorname{Ext}_{R}{ }^{1}(K, X)=0$.

(i) $\Rightarrow$ (ii): Jeder Untermodul $U$ von $M$ hat die Eigenschaft (E), ist also nach (2.1) kotorsion. 
(ii) $\Rightarrow$ (iii): Weil insbesondere $T(M) / D(T(M))$ kotorsion ist, ist es nach [11, Corollary 7.8] beschränkt, also $M=M_{1} \oplus D \oplus B$ mit $M_{1}$ torsionsfrei, $D$ torsionsvoll und teilbar, $B$ beschränkt. Weil $R^{(\mathrm{N})}$ nicht kotorsion ist, hat $M_{1}$ endlichen Rang, und weil jeder reduzierte Untermodul von $D$ wieder beschränkt ist, ist $D$ bereits artinsch. Ist nun $R$ nicht-lokal oder lokal und unvollständig, so ist $R_{R}$ nicht kotorsion [11, Corollary 7.9], also $M_{1}=0$, und wir sind fertig. Ist aber $R$ lokal und vollständig, so ist $M_{1}$ nach (2.2) linear-kompakt, und es folgt die Behauptung.

(iii) $\Rightarrow$ (i): Nach Voraussetzung gibt es ein $0 \neq r \in R$, so daß $M r$ linear-kompakt ist. In der exakten Folge

$$
0 \rightarrow M r \subset M \rightarrow M / M r \rightarrow 0
$$

hat also das erste und dritte Glied die Eigenschaft (EE), also auch $M$.

Bemerkung. Über einem nicht-lokalen Dedekindring ist nach (1.1 Folgerung) jeder linear-kompakte Modul bereits artinsch. Daher ist in diesem Fall (iii) äquivalent mit (iii'): Der divisible Anteil von $M$ ist artinsch, und der reduzierte Anteil beschränkt. Nach [15, Theorem 3.1] ist das noch äquivalent damit, daß $M$ komplementiert ist und fast alle Primärkomponenten von $M$ gleich Null sind.

\section{Radikal-komplementierte Moduln über einem diskreten Bewertungsring.}

In diesem Abschnitt ist $R$ stets ein diskreter Bewertungsring mit Quotientenkörper $K \neq R$ und dem maximalen Ideal $(p)$. In [15] wurde gezeigt, daß ein $R$-Modul mit kleinem Radikal bereits koatomar ist, d.h. keine radikalvollen Faktoren hat, und dann schon direkte Summe aus einem endlich erzeugten freien und einem beschränkten Modul ist. Es soll nun folgende schwächere Eigenschaft untersucht werden, die eng mit der Eigenschaft (E) zusammenhängt:

Definition. Ein Modul $M$ heißt radikal-komplementiert, wenn $\operatorname{Ra}(M)$ ein Komplement in $M$ hat.

Satz 3.1. Für einen $R$-Modul $M$ sind äquivalent:

(i) $M$ ist radikal-komplementiert.

(ii) Es gibt ein $n \geqq 0$ mit $\mathrm{Ra}^{n}(M) / \mathrm{Ra}^{n+1}(M)$ endlich erzeugt.

(iii) "Der" Basis-Untermodul von $M$ is koatomar.

(iv) $E s$ ist $M=T(M) \oplus X$, wobei der reduzierte Anteil von $T(M)$ beschränkt ist, und $X / \operatorname{Ra}(X)$ endlich erzeugt. 
BeweIs. (i) $\Rightarrow$ (ii): Sei $V$ ein Komplement von $\operatorname{Ra}(M)$ in $M$. Als wesentliche Überdeckung von $M / \operatorname{Ra}(M)$ ist auch $V$ koatomar, also $V=F \oplus B$ mit $F$ endlich erzeugt und frei, $B p^{n}=0$ für ein $n \geqq 0$. Aus $V p^{n}+M p^{n+1}=M p^{n}$ erhält man den Epimorphismus

also die Behauptung.

$$
F p^{n} \rightarrow M p^{n} / M p^{n+1},
$$

(ii) $\Rightarrow$ (iii): Sei $S$ ein Basis-Untermodul von $M$, und $S=\oplus_{i \in I} S_{i}$ mit $S_{i}$ zyklisch. Weil nun

$$
S p^{n} / S p^{n+1} \cong M p^{n} / M p^{n+1}
$$

endlich erzeugt ist, gilt für fast alle $i \in I$, daß $S_{i} p^{n}=S_{i} p^{n+1}$, also $S_{i} p^{n}=0$, so daß $S$ direkte Summe aus endlich vielen zyklischen und einem beschränkten Modul ist, also koatomar.

(iii) $\Rightarrow$ (i): Jeder Basis-Untermodul $S$ von $M$ ist ein Komplement des Radikals, denn klar ist $S+\operatorname{Ra}(M)=M$, und weil $S$ koatomar ist, hat man auch noch $S \cap \operatorname{Ra}(M)=\operatorname{Ra}(S)$ klein in $S$.

(iii) $\Rightarrow$ (iv): Auch der Basis-Untermodul von $T(M)$ ist koatomar, also beschränkt, so daß $T(M) / D(T(M))$ direkter Summand in $M / D(T(M))$ ist. Es folgt $M=T(M) \oplus X$, und weil auch im torsionsfreien $X$ der BasisUntermodul koatomar, also endlich erzeugt ist, folgt die Behauptung.

(iv) $\Rightarrow$ (iii): Nach Voraussetzung sind die Basis-Untermoduln von $T(M)$ und $X$ koatomar, also auch der von $M$.

Man zeigt leicht, daß die Bedingung (iii) noch äquivalent damit ist, daß zwei vergleichbare Basis-Untermoduln von $M$ stets schon übereinstimmen. Aus den Äquivalenzen von (3.1) folgt auch unmittelbar:

Lemma 3.2. (a) Die Klasse der radikal-komplementierten $R$-Moduln ist gegenüber Faktormoduln, reinen Untermoduln und Gruppenerweiterungen abgeschlossen.

(b) Ist $M$ radikal-komplementiert und $M / U$ reduziert, so ist auch $U$ radikal-komplementiert.

(c) Genau dann ist jeder Untermodul von $M$ radikal-komplementiert, wenn $T(M)$ komplementiert ist und $M / T(M)$ endlichen Rang hat.

Lemma 3.3. Für einen $R$-Modul sind $M$ äquivalent:

(i) $M$ ist radikal-komplementiert.

(ii) $M$ hat in seiner injektiven Hülle ein Komplement.

(iii) $Z u$ jeder Erweiterung $M \subset N$ gibt es ein $X \subset N$, mit $X+M=N$ und $X \cap M$ klein in $N$.

(iv) $M$ hat in jeder Erweiterung $N$, mit N/M koatomar, ein Komplement. 
BeweIs. Vorbemerkung: Ein Untermodul $A$ von $B$ ist genau dann klein in $B$, wenn $A$ koatomar ist und enthalten in $\operatorname{Ra}(B)$. Ist $A$ nur koatomar, so braucht es natürlich nicht klein in $B$ zu sein, aber aus

$$
X / \operatorname{Ra}(B) \oplus(A+\operatorname{Ra}(B)) / \operatorname{Ra}(B)=B / \operatorname{Ra}(B)
$$

folgt immerhin noch $X+A=B$ mit $X \cap A$ klein in $B$.

(i) $\Rightarrow$ (ii) : Ist $M \subset \widehat{M}$ eine injektive Hülle, so folgt nach [15, Lemma 2.3], daß $M$ in $\widehat{M}$ ein Komplement hat.

(ii) $\Rightarrow$ (iii): Ist $M \subset N$, und $N \subset \hat{N}$ eine injektive Hülle, so kann man $M \subset \widehat{M} \subset \widehat{N}$ wählen, und ist nach Voraussetzung $V$ ein Komplement von $M$ in $\widehat{M}$, weiter $W \oplus \widehat{M}=\widehat{N}$, so ist $V+W$ ein Komplement von $M$ in $\widehat{N}$. Mit $V_{1}=(V+W) \cap N$ erhält man insbesondere $V_{1}+M=N, V_{1} \cap M$ koatomar. Nach der Vorbemerkung gibt es nun ein $X \subset V_{1} \operatorname{mit} X+\left(V_{1} \cap M\right)=$ $V_{1}$ und $X \cap\left(V_{1} \cap M\right)$ klein in $V_{1}$, sodaß endlich $X+M=N$ und $X \cap M$ klein in $N$ ist.

(iii) $\Rightarrow$ (iv): Sei $M \subset N$ und $X$ wie vorausgesetzt. Ist nun $N / M \cong$ $X / X \cap M$ koatomar, so ist mit $X \cap M$ auch $X$ koatomar, also komplementiert, und ist jetzt $V$ ein Komplement von $X \cap M$ in $X$, so ist $V$ auch ein Komplement von $M$ in $N$.

(iv) $\Rightarrow$ (i): Die Eigenschaft (iv) vererbt sich nach $(1.3, b)$ auf Faktormoduln (denn im dortigen Diagramm ist $\mathrm{Kok} h \cong N /(B / A)$ ), insbesondere auf $\operatorname{Ra}(M)$. Damit hat $\operatorname{Ra}(M)$ ein Komplement in $M$.

Bemerkung. Aus (ii) folgt nicht, daß $M$ in jeder wesentlichen Erweiterung ein Komplement hat: Sei $R$ unvollständig, $N$ ein torsionsfreier, direkt unzerlegbarer $R$-Modul vom Rang 2 (siehe [6, Theorem 19]), $S$ ein Basis-Untermodul von $N$, und $S \subsetneq M \subsetneq N$. Dann ist $M \cong R^{2}$ und $N / M$ torsionsvoll, aber $M$ hat kein Komplement in $N$. - Andrerseits kann man das Ergebnis in (ii) $\Rightarrow$ (iii), daß $M$ in jeder injektiven Erweiterung $N$ ein Komplement hat, noch verbessern: Es genügt zu verlangen, daß in $N / T(N)$ jeder reine Untermodul schon direkter Summand ist. Solche Moduln nannten wir in [15, Satz 2.7] reinzerfallend, und es wurde dort gezeigt, daß das äquivalent damit ist, daß der reduzierte Anteil koatomar ist. Insbesondere ist die Klasse der reinzerfallenden $R$-Moduln gegenüber Faktormoduln und endlichen direkten Summen abgeschlossen.

Satz 3.4. Ist $M$ radikal-komplementiert, so hat $M$ in jeder Erweiterung $N$, mit $N / T(N)$ reinzerfallend, ein Komplement.

BzWHIs. (1) Sei zunächst spezieller $M$ koatomar: Dann gibt es nach 
der Vorbemerkung in (3.3) ein $X \subset N$ mit $X+M=N$ und $X \cap M$ klein in $N$. Die Menge

$$
\{V \mid V \subset X \text { und } \operatorname{Ra}(V)=V \cap \operatorname{Ra}(N)\}
$$

hat nach Zorn ein maximales Element $V_{0}$, und wir behaupten, daß $V_{0}$ ein Komplement von $M$ in $N$ ist: Weil $V_{0} \cap M$ klein in $N$, also auch klein in $V_{0}$ ist, bleibt nur noch $V_{0}+M=N$ zu zeigen. Nun hat in $\bar{N}=N / V_{0}$ der Untermodul $\bar{X}$, wegen der Maximalität von $V_{0}$, folgende Eigenschaft: Ist $A \subset \bar{X}$ mit $\operatorname{Ra}(A)=A \cap \operatorname{Ra}(\bar{N})$, so ist $A$ schon Null. Daraus folgt, daß $\bar{X}$ reduziert ist, und $\bar{X} \subset \operatorname{Ra}(\bar{N})$. Aus $\bar{M}+\bar{X}=\bar{N}$ folgt jetzt, weil $\bar{M}$ koatomar ist, daß $\bar{N}$ reduziert und radikal-komplementiert ist, also $\bar{N}=$ $H_{1} \oplus H_{2}$ mit $H_{1}$ torsionsfrei und $H_{2}$ beschränkt. Als Faktor von $N / T(N)$ ist nun $H_{1}$ reinzerfallend, also sogar endlich erzeugt. Damit ist $\bar{N}$ koatomar, und $\bar{X}$ klein in $\bar{N}$. Es folgt $\bar{M}=\bar{N}$, d.h. $V_{0}+M=N$ wie behauptet.

(2) Sei nun $M$ radikal-komplementiert, $S$ ein Basis-Untermodul von $M$, und $V / S \oplus M / S=N / S$ mit $S \subset V \subset N$. Weil dann $V$ rein in $N$, also auch $V / T(V)$ reinzerfallend ist, gibt es nach (1) ein Komplement $W$ von $S$ in $V$, und $W$ ist dann auch ein Komplement von $M$ in $N$.

Folgerdng 1. Ist $M / \operatorname{Ra}(M)$ einfach, so hat $M$ in jeder Erweiterung $N$, mit $N / M$ torsionsvoll, ein Komplement.

Folgerung 2. Ist $M / \operatorname{Ra}(M)$ einfach, so hat $M$ in jeder Erweiterung $N$, mit $M$ nicht rein in $N$, ein Komplement.

Folgerung 3. Ist $R$ vollständig, so hat jeder radikal-komplementierte $R$-Modul die Eigenschaft (E).

Folgerung 4. Ist $R$ vollständig, und $A \subset B \subset C$, so gilt: Hat $A$ ein Komplement in $C$, so hat $A$ auch ein Komplement in $B$.

Folgerung 5. Ist $R$ vollständig, und $X+M=N$ mit $X \cap M$ klein in $N$, so gibt es ein Komplement $V$ von $M$ in $N$, mit $V \subset X$.

Bwwris. (1) Wählt man einen Basis-Untermodul $S$ von $M$ und $V / S \oplus M / S=N / S$, so ist $V / S$ torsionsvoll, und aus der Voraussetzung über $M$ folgt, daß $S$ zyklisch ist, also $\operatorname{Rang}(V / T(V)) \leqq 1$. Nach dem Satz hat nun $S$ ein Komplement in $V$, und man ist fertig.

(2) Seien $S$ und $V$ wie eben, außerdem der Zwischenmodul $V^{\prime}$ definiert durch $V^{\prime} / S=T(V / S)$. Nach (1) hat nun $S$ ein Komplement $X$ in $V^{\prime}$, und weil nach Voraussetzung $M$ nicht rein in $N$, also auch $S$ nicht rein in $V^{\prime}$ 
ist, folgt $X \cap S \neq 0$, sodaß $S / X \cap S \cong V^{\prime} \mid X$ kotorsion ist. Wählt man nun $Y\left|X \oplus V^{\prime}\right| X=V \mid X$ mit $X \subset Y \subset V$, so ist $Y$ ein Komplement von $S$ in $V$, denn die Summe ist klar, und $Y \cap S=Y \cap V^{\prime} \cap S=X \cap S$ ist klein in $X$, also erst recht klein in $Y$.

(3) Weil $R$ vollständig, ist jeder reduzierte radikal-komplementierte $R$-Modul nach [6, Theorem 23] bereits koatomar. Damit ist jetzt im Beweis des Satzes die Voraussetzung " $N / T(N)$ reinzerfallend« überflüssig.

(4) Es genügt, daß es ein $X \subset C$ gibt mit $X+A=C, X \cap A$ klein in $C$. Dann folgt nämlich $(X \cap B)+A=B$, und das koatomare $X \cap A$ hat nach eben ein Komplement in $X \cap B$, das dann auch ein Komplement von $A$ in $B$ ist.

(5) Dies wurde soeben gezeigt mit $A=M$ und $B=C=N$.

Für einen radikal-komplementierten $R$-Modul $M$ läßt sich die Struktur von $M^{*}=\operatorname{Ext}_{R}{ }^{1}(K / R, M)$ nach $(3.1, \mathrm{iv})$ sofort angeben. Weil andrerseits $R^{*}$ nach (3.4 Folgerung 2) die Eigenschaft (E) hat, haben wir als Hauptergebnis dieses Abschnittes:

Theorem 3.5. Für einen $R$-Modul $M$ sind äquivalent:

(i) $M$ hat die Eigenschaft (E).

(ii) $M$ ist radikal-komplementiert und kotorsion.

(iii) $M \cong\left(R^{*}\right)^{a} \times D \times B$, wobei $R^{*}$ die Vervollständigung von $R$ ist, $a \geqq 0$, $D$ teilbar und $B$ beschränkt.

Zum Abschluß dieses Paragraphen wollen wir auch die reinzerfallenden $R$-Moduln durch eine Komplement-Eigenschaft des Radikals bzw. der injektiven Hülle charakterisieren:

Satz 3.6. Für einen $R$-Modul $M$ sind äquivalent:

(i) $M$ hat in seiner injektiven Hülle ein starkes Komplement.

(ii) $\mathrm{Ra}(M)$ hat ein starkes Komplement in $M$.

(iii) Der reduzierte Anteil von $M$ ist koatomar.

In diesem Fall sind die Basis-Untermoduln von $M$ genau die starken Komplemente des Radikals.

Bewers. Ein Komplement $V$ von $U$ in $M$ heißt nach [16] starkes Komplement, wenn zusätzlich $V \cap U$ direkter Summand in $U$ ist, d.h. wenn es ein $U^{\prime} \subset U$ gibt mit $V \oplus U^{\prime}=M$.

(i) $\Rightarrow$ (ii): Sei $M \subset \widehat{M}$ eine injektive Hülle, und nach Voraussetzung $V$ ein starkes Komplement von $M$ in $\widehat{M}$. Aus $M^{\prime} \subset M$ mit $V \oplus M^{\prime}=\widehat{M}$ folgt 
$(V \cap M) \oplus M^{\prime}=M$ mit $V \cap M$ koatomar und $M^{\prime}$ teilbar. Damit ist $V \cap M$ bereits ein starkes Komplement von $\operatorname{Ra}(M)$ in $M$.

(ii) $\Rightarrow$ (iii): Ist $V$ ein starkes Komplement von $\mathrm{Ra}(M)$ in $M$, und $X \subset \operatorname{Ra}(M)$ mit $V \oplus X=M$, so ist $V$ koatomar und $X$ teilbar, also $X=$ $D(M)$, und damit $M / D(M)$ koatomar.

(iii) $\Rightarrow$ (i): Sei $M \subset \widehat{M}$ eine injektive Hülle, und $V \oplus D(M)=\widehat{M}$. Wir behaupten, daß $V$ bereits ein starkes Komplement von $M$ in $\widehat{M}$ ist, und dazu ist nur mehr zu zeigen, daß in $V$ der Untermodul $V \cap M$ klein ist, d.h. koatomar. Wegen $V \cap M \cong M / D(M)$ ist das aber gerade die Voraussetzung.

Aus diesem Beweis folgt auch der Zusatz über die Basis-Untermoduln.

Endlich soll noch bemerkt werden, daß ein $R$-Modul $M$ genau dann in jeder Erweiterung ein starkes Komplement hat, wenn der reduzierte Anteil von $M$ halbeinfach ist, und bekanntlich ist das äquivalent damit, daß $M$ neat-injektiv ist, d.h. in jeder Erweiterung $N$, mit $\operatorname{Ra}(M)=$ $M \cap \operatorname{Ra}(N)$, direkter Summand ist.

\section{Das Radikal von $\boldsymbol{\Sigma}$-selbstprojektiven Moduln.}

Es soll über einem beliebigen Ring $R$ für spezielle Moduln $M_{R}$ untersucht werden, wann aus der Tatsache, daß $\operatorname{Ra}(M)$ ein Komplement in $M$ hat, bereits die Kleinheit des Radikals folgt. Dazu sei an folgenden Begriff erinnert: Ein Modul $M$ heißt $\Sigma$-selbstprojektiv (siehe [4]), wenn für jede Indexmenge $I$ der Modul $M^{(I)}$ selbstprojektiv ist.

Satz 4.1. Ist der Modul $M$ $\Sigma$-selbstprojektiv und $U \subset \operatorname{Ra}(M)$, so gilt: $H$ at $U$ ein Komplement in $M$, so ist $U$ bereits klein in $M$.

BEwEIs. (1) Es wird eine Verallgemeinerung von "das Radikal ist klein" benötigt, die bei Bildung von direkten Summen erhalten bleibt: Ein Modul $M$ heiße deformierbar, wenn es zu jedem $m \in \operatorname{Ra}(M)$ ein $\mu \in \operatorname{End}(M)$ gibt mit $\mu(m)=m$ und $\mu(\operatorname{Ra}(M))$ klein in $M$. Dafür zeigt man leicht, daß $M=\oplus M_{i}$ genau dann deformierbar ist, wenn alle $M_{i}$ deformierbar sind.

(2) Ist $M \sum$-selbstprojektiv und $\alpha \in \operatorname{End}(M)$ mit $\operatorname{Bi}(1-\alpha) \subset \operatorname{Ra}(M)$, so ist $\alpha$ ein Monomorphismus (für projektives $M$ ist das Theorem 3 in [2]): Zunächst ist $M$ deformierbar nach (1), denn zu einem Erzeugendensystem $\left(m_{i} \mid i \in I\right)$ zerfällt der kanonische Epimorphismus

$$
\coprod_{i \in I} m_{i} R \rightarrow M,
$$


so daß $M$ bis auf Isomorphie direkter Summand einer direkten Summe von Zyklischen ist. Ist nun $\alpha$ wie angegeben und $m \in \operatorname{Ke} \alpha$, so gibt es ein $\mu \in \operatorname{End}(M)$ mit $\mu(m)=m$ und $\mu(\operatorname{Ra}(M))$ klein in $M$. Definiert man nun $\beta=1-\mu(1-\alpha)$, so erhält man $m \in \operatorname{Ke} \beta$ und $\operatorname{Bi}(1-\beta)$ klein in $M$, so daß $1-\beta$ im Radikal von $\operatorname{End}(M)$ liegt (weil $M$ selbstprojektiv), also $\beta$ ein Isomorphismus ist. Es folgt $m=0$.

(3) Sei nun $M \Sigma$-selbstprojektiv, $U \subset \mathrm{Ra}(M)$ und $V$ ein Komplement von $U$ in $M:$ Dann gibt es ein $\alpha \in \operatorname{End}(M)$ mit $\operatorname{Bi} \alpha \subset V$ und $\operatorname{Bi}(1-\alpha) \subset U$, so daß aus $\alpha(V)+U=M$ weiter folgt $\alpha(V)=V$, also $M=\alpha^{-1}(V)=\alpha^{-1} \alpha(V)$ $=V+\operatorname{Ke} \alpha=V$, denn nach (2) ist $\alpha$ ein Monomorphismus. Damit ist $U$ klein in $M$.

Folgerung (W. Zimmermann). Ein $\Sigma$-selbstprojektiver, radikalvoller Modul ist bereits Null.

Bemerkung. Über einem vollständigen diskreten Bewertungsring $R$ gilt sogar für jeden reduzierten $R$-Modul $M$ : Ist $U \subset \mathrm{Ra}(M)$ und hat $U$ ein Komplement in $M$, so ist $U$ bereits klein in $M$.

\section{Die Moduln mit der Eigenschaft (E) über einem nicht-lokalen Dedekindring.}

In diesem Abschnitt ist $R$ stets ein Dedekindring, kein Körper. Obwohl sich in (5.2 Folgerung 1) ein wesentlicher Unterschied zum lokalen Fall des Abschnittes 3 ergibt, lassen sich die Resultate mit ähnlichen Methoden wie zuvor herleiten, so daß wir die Beweise von (5.3) bis (5.6) dem Leser überlassen.

HIIfssatz 5.1. Sei $A \subset B \subset C$ und besitze $A$ ein Komplement in $C$.

Ist dann entweder $B$ torsionsvoll oder $B / A \supset \operatorname{So}(C / A)$, so hat $A$ auch ein Komplement in $B$.

Bewers. (1) Sei zunächst spezieller $A$ klein in $C$. Dann ist $A$ koatomar, also im ersten Fall in jeder Primärkomponente beschränkt, so daß $A$ im torsionsvollen $B$ ein Komplement hat. Im zweiten Fall ist $A$ sogar klein in $B$, denn mit

$$
\mathfrak{p}^{-1} c(A)=\{c \in C \mid \mathfrak{c p} \subset A\}
$$

folgt aus der Voraussetzung $\mathfrak{p}^{-1} C(A) \subset B$ für alle maximalen Ideale $\mathfrak{p}$, also

$$
A=A \cap C \mathfrak{p}=\left(\mathfrak{p}^{-1} c(A)\right) \mathfrak{p} \subset B \mathfrak{p}
$$

für alle $\mathfrak{p}$, also $A \subset \operatorname{Ra}(B)$. 
(2) Ist nun $V$ ein Komplement von $A$ in $C$, so folgt $V \cap A \subset V \cap B \subset V$ und entweder $V \cap B$ torsionsvoll oder

$$
V \cap B / V \cap A \supset \operatorname{So}(V / V \cap A) .
$$

Damit hat jetzt $V \cap A$ ein Komplement $W$ in $V \cap B$, und $W$ ist dann auch ein Komplement von $A$ in $B$.

FolgerUng. Ein $R$-Modul $M$ hat genau dann die Eigenschaft (E), wenn er in jeder Erweiterung $N$, mit $N / M$ teilbar, ein Komplement hat.

BeisPiele über $R=Z$. (1) Sei $B=\Pi_{p}(Z /(p))$, wobei $p$ alle Primzahlen durchläuft, $A=T(B)$ und $C$ eine injektive Erweiterung von $B$. Dann hat $A$ ein Komplement in $C$, aber keines in $B$.

(2) Sei $Z \subsetneq B \subset Q$. Hat dann $Z$ ein Komplement in $B$, so ist $Z$ schon klein in $B$, und das ist äquivalent damit, daß $B$ alle rationalen Zahlen mit quadratfreiem Nenner enthält.

Während jede wesentliche Überdeckung eines torsionsfreien $R$-Moduls schon ein Isomorphismus ist, braucht eine wesentliche Überdeckung eines Torsionsmoduls nicht einmal torsionsvoll zu sein (siehe Beispiel 2). Man hat jedoch:

HILFSSATZ 5.2. Ist $f: A \rightarrow B$ ein wesentlicher Epimorphismus, und ist in $B$ mindestens eine Primärkomponente gleich Null, so gilt:

(a) Kef ist torsionsvoll.

(b) In

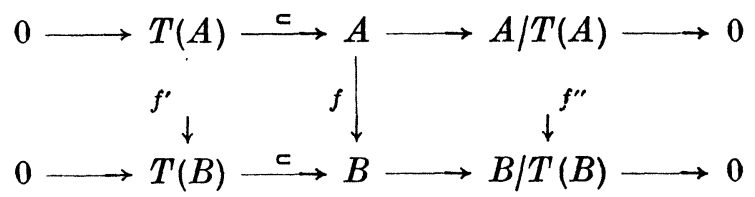

ist $f^{\prime}$ wieder ein wesentlicher Epimorphismus, und $f^{\prime \prime}$ ein Isomorphismus.

(c) Die injektive Hülle von $A$ ist isomorph zur injektiven Hülle von $B$.

BeweIs. (a) Sei $T_{\mathfrak{p}}(B)$, die $\mathfrak{p}$-Komponente von $B$, gleich Null für ein maximales Ideal $\mathfrak{p}$. Dann ist $X=\operatorname{Ke} f \mathfrak{p}$-teilbar, denn mit

$$
\mathfrak{p}^{-1}{ }_{A}(X)=\{a \in A \mid a \mathfrak{p} \subset X\}
$$

ist $\mathfrak{p}^{-1}{ }_{A}(X) / X \subset T_{\mathfrak{p}}(A / X)=0$, also

$$
X \mathfrak{p}=\left(\mathfrak{p}^{-1}{ }_{A}(X)\right) \mathfrak{p}=X \cap A \mathfrak{p}=X .
$$


Weil aber $X$ koatomar, ist jetzt jeder $\mathfrak{p}$-primäre Faktor von $X$ gleich Null, insbesondere

$$
\operatorname{Hom}_{R}\left(X, T_{\mathfrak{p}}(K / R)\right)=0 .
$$

Daraus folgt aber $\operatorname{Hom}_{R}(X, K)=0$, also $X=\operatorname{Ke} f$ torsionsvoll wie behauptet.

(b) Aus $\operatorname{Ke} f \subset T(A)$ folgt sofort die Isomorphie von $f^{\prime \prime}$, und die Surjektivität von $f^{\prime}$. Es ist aber auch $\operatorname{Ke} f^{\prime}$ klein in $T(A)$, denn $\operatorname{Ke} f^{\prime}=\operatorname{Ke} f$ ist koatomar, und enthalten in $\operatorname{Ra}(T(A))$.

(c) Sei $X=\operatorname{Ke} f$, außerdem $A \subset \hat{A}$ eine injektive Hülle, und $X \subset \hat{X} \subset \hat{A}$. Weil nun $X$ torsionsvoll und koatomar, also direkte Summe von zyklischen Torsionsmoduln ist, hat $\operatorname{man} \hat{X} / X \cong \hat{X}$, also $\hat{A} \cong \hat{A} / X$. Weiter folgt aus $X \subset \operatorname{Ra}(A)$, in vollkommener Dualität zum ersten Beweisschritt von (5.1), daß $A / X$ groß in $\hat{A} / X$ ist, also $\hat{A} / X \cong \hat{B}$.

Folgerung 1. Ist $R$ nicht-lokal, so ist jede wesentliche Überdeckung eines $\mathfrak{p}$-primären (und beschränkten) $R$-Moduls wieder $\mathfrak{p}$-primär (und beschränkt).

Folgerung 2. Hat $R$ unendlich viele maximale Ideale, so ist jede wesentliche Überdeckung eines artinschen $R$-Moduls wieder artinsch.

SATZ 5.3. Ist $R$ nicht-lokal und $\mathfrak{p}$ ein maximales Ideal, so sind für einen $R$-Modul $M$ äquivalent:

(i) $M \mathfrak{p}$ hat ein Komplement in $M$.

(ii) Die Folge $M \supset M \mathfrak{p} \supset M_{\mathfrak{p}}{ }^{2} \supset \ldots$ ist stationär.

(iii) Es ist $M=T_{\mathfrak{p}}(M) \oplus X$, wobei $T_{\mathfrak{p}}(M)$ radikal-komplementiert ist, und $X \mathfrak{p}$-teilbar.

(iv) $M$ hat in jeder Erweiterung $N$, mit $N / M$ p-primär, ein Komplement.

In diesem Fall sagen wir, daß $M$ die Eigenschaft $\left(\mathrm{E}^{\mathfrak{p}}\right)$ hat.

Über $R=Z$ hat man noch den von Fuchs (siehe [ 3 § 32]) eingeführten Begriff einer $p$-Basis-Untergruppe $B$ von $M$. Bemerkt man, daß für ein Komplement $V$ von $B$ notwendig $V=\bigcap_{i=1}^{\infty} M p^{i}$ und $V \oplus B=M$ gilt, daß außerdem für jede Primzahl $q \neq p$ auch $B q$ eine $p$-Basis-Untergruppe von $M$ ist, so erhält man folgende Fortsetzung von (5.3):

SATz 5.3*. Für eine abelsche Gruppe $M$ ist $\left(\mathrm{E}^{p}\right)$ weiter äquivalent mit:

(a) "Die" $p$-Basis-Untergruppe von $M$ ist beschränkt.

(b) Jede $p$-Basis-Untergruppe von $M$ hat ein Komplement in $M$.

(c) Zwei vergleichbare $p$-Basis-Untergruppen von $M$ stimmen stets schon überein. 
In diesem Fall sind die $p$-Basis-Untergruppen von $M$ genau die starken Komplemente von $M p$ in $M$.

LeMma 5.4. Ist $R$ nicht-lokal und $\mathfrak{p}$ ein maximales Ideal, so gilt:

(a) Die Klasse der R-Moduln mit der Eigenschaft $\left(\mathrm{E}^{\mathfrak{p}}\right)$ ist gegenüber Faktormoduln, reinen Untermoduln und Gruppenerweiterungen abgeschlossen.

(b) Hat $M$ die Eigenschaft $\left(\mathrm{E}^{\mathfrak{p}}\right)$ und ist $M / U$ reduziert, so hat auch $U$ die Eigenschaft $\left(\mathrm{E}^{\mathfrak{p}}\right)$.

(c) Genau dann hat jeder Untermodul von $M$ die Eigenschaft $\left(\mathbf{E}^{p}\right)$, wenn $M$ torsionsvoll ist und die $\mathfrak{p}$-Komponente von $M$ komplementiert ist.

Auch die folgende "Globalisierung « bereitet keine Schwierigkeiten, wenn man beim Schritt (ii) $\Rightarrow$ (iii) eine injektive Hülle $\widehat{M}$ und den Zwischenmodul $N=\mathfrak{p}^{-\mathbf{1}} \hat{M}(M)$ wählt:

Lemma 5.5. Ist $R$ nicht-lokal, so sind für einen $R$-Modul $M$ äquivalent:

(i) $M$ hat in jeder Erweiterung $N$, mit $N / M$ torsionsvoll, ein Komplement.

(ii) $M$ hat in jeder wesentlichen Erweiterung ein Komplement.

(iii) $M$ hat die Eigenschaft $\left(\mathrm{E}^{\mathfrak{p}}\right)$, für jedes maximale Ideal $\mathfrak{p}$.

(iv) $T(M)$ ist radikal-komplementiert, und $M / T(M)$ teilbar.

Aus der letzten Äquivalenz bekommt man jetzt durch den Funktor $\operatorname{Ext}_{R}{ }^{1}(K / R,-)$ die Struktur der $R$-Moduln mit der Eigenschaft (E). Weil andrerseits sich jede Erweiterung $M \subset N$ trivialerweise in $M \subset N^{\prime} \subset N$ aufteilen läßt mit $N^{\prime} / M$ torsionsvoll und $N / N^{\prime}$ torsionsfrei, ist im Folgenden auch der Schritt (ii) $\Rightarrow$ (i) klar, so daß wir als Hauptergebnis dieses Abschnittes erhalten:

THEOREM 5.6. Ist $R$ nicht-lokal, so sind für einen $R$-Modul $M$ äquivalent:

(i) $M$ hat die Eigenschaft (E).

(ii) $M$ ist kotorsion, und $M$ hat in jeder Erweiterung $N$, mit $N / M$ torsionsvoll, ein Komplement.

(iii) $M \cong D \times\left(\Pi_{\mathfrak{p}} A_{\mathfrak{p}}\right)$, wobei $D$ teilbar ist, $\mathfrak{p}$ alle maximalen Ideale von $R$ durchläuft, und jedes $A_{\mathfrak{p}} \mathfrak{p}$-primär und beschränkt ist.

Folgerung. Hat $M$ die Eigenschaft (E), und ist $M / U$ reduziert, so hat auch $U$ die Eigenschaft (E). 
Im Fall unendlich vieler maximaler Ideale (aber auch über einem unvollständigen diskreten Bewertungsring) tritt bei der Eigenschaft (E) noch ein Phänomen auf, das wir für $R=Z$ erläutern wollen: In der abelschen Gruppe

$$
N=\Pi_{p}\left(\mathrm{Z} /\left(p^{2}\right)\right)
$$

hat die Untergruppe $M=\operatorname{Ra}(N)$ wieder die Eigenschaft (E). Insbesondere hat $M$ ein Komplement $V$ in $N$, aber $V$ selbst hat kein Komplement mehr in $N$, denn $M$ ist reduziert und nicht klein in $N$. Will man das vermeiden, so erhält man folgende Klasse von $R$-Moduln:

Satz 5.7. Ist $R$ nicht-lokal, so sind für einen $R$-Modul $M$ äquivalent:

(i) Ist $M \subset N$, so gibt es ein Komplement $V$ von $M$ in $N$ derart, daß auch noch $V$ ein Komplement in $N$ hat.

(ii) Der reduzierte Anteil von $M$ ist beschränkt.

Falls $R$ unendlich viele maximale Ideale hat, ist das weiter äquivalent mit

(iii) $M$ hat in seiner injektiven Hülle ein Komplement, und $M$ ist $\mathfrak{p}$-teilbar für fast alle maximalen Ideale $\mathfrak{p}$.

Bewkis. (i) $\Rightarrow$ (ii): Weil $M$ insbesondere die Eigenschaft (E) hat, ist der reduzierte Anteil nach (5.6) von der Form $\Pi_{\mathfrak{p}} A_{\mathfrak{p}}$ mit $A_{\mathfrak{p}}$ beschränkt, und wir müssen nur noch zeigen, daß fast alle $A_{\mathfrak{p}}$ gleich Null sind, d.h. $M / D(M)$ koatomar ist: Dazu sei $M \subset \widehat{M}$ eine injektive Hülle, und $M \subset N \subset \hat{M}$ definiert durch $N / M=\operatorname{So}(\widehat{M} / M)$. Nach Voraussetzung hat man ein Komplement $V$ von $M$ in $N$, und ein Komplement $W$ von $V$ in $N$. Weil $M=\operatorname{Ra}(N)$, ist $N / V$ radikalvoll, also auch $W$, und aus $W \subset$ $D(M)$ erhält man den Epimorphismus $V \cap M \rightarrow M / D(M)$ mit $V \cap M$ koatomar, also die Behauptung.

(ii) $\Rightarrow$ (i): Sei $M=D \oplus A$ mit $D$ teilbar und $A$ beschränkt. Ist dann $M \subset N$, so gibt es ein $D \subset U \subset N$ mit $U / D$ Komplement von $M / D$ in $N / D$. Weil aber $M / D$ komplementiert ist, gibt es auch noch ein $D \subset W \subset M$ mit $W / D$ Komplement von $U / D$ in $N / D$. Ist nun $V \oplus D=U$, so zeigt man leicht, daß $V$ ein Komplement von $M$ in $N$ ist, und $W$ ein Komplement von $V$ in $N$.

(ii) $\Rightarrow$ (iii): $M$ hat ja sogar die Eigenschaft (E), und die Teilbarkeit ist klar.

(iii) $\Rightarrow$ (ii): Aus der Teilbarkeitsbedingung folgt, daß in $\widehat{M} / M$ fast alle Primärkomponenten Null sind. Ist nun $V$ ein Komplement von $M$ in $\widehat{M}$, so ist $V \cap M$ als Kern des wesentlichen Epimorphismus $V \rightarrow \widehat{M} / M$ nach (5.2) beschränkt. Sei jetzt $M^{\prime}$ ein Komplement von $V \cap M$ in $M$. 
Dann ist $M^{\prime}$ auch ein Komplement von $V$ in $\widehat{M}$, also $M^{\prime}$ teilbar, also $M^{\prime} \subset D(M)$. Damit erhält man den Epimorphismus $V \cap M \rightarrow M / D(M)$, so daß auch $M / D(M)$ beschränkt ist.

\section{LITERATUR}

1. H. Bass, Finitistic dimension and a homological generalization of semi-primary rings, Trans. Amer. Math. Soc. 95 (1960), 466-488.

2. I. Beck, Projektive and free modules, Math. Z. 129 (1972), 231-234.

3. L. Fuchs, Infinite abelian groups I, Academic Press, New York, London, 1970.

4. K. R. Fuller and D. A. Hill, On quasi-projective modules via relative projectivity, Arch. Math. (Basel) 21 (1970), 369-373.

5. J. S. Golan, Quasi perfect modules, Quart. J. Math. Oxford Ser. (2), 22 (1971), 173-182.

6. I. Kaplansky, Infinite abelian groups, University of Michigan Press, Ann Arbor, Michigan, 1969.

7. F. Kasch und E. A. Mares, Eine Kennzeichnung semiperfekter Moduln, Nagoya Math. J. 27 (1966), 525-529.

8. E. A. Mares, Semiperfect modules, Math. Z. 82 (1963), 347-360.

9. E. Matlis, Divisible modules, Proc. Amer. Math. Soc. 11 (1960), 385-391.

10. E. Matlis, Some properties of noetherian domains of dimension one, Canad. J. Math. 13 (1961), 569-586.

11. R. J. Nunke, Modules of extensions over Dedekind rings, Illinois J. Math. 3 (1959), 222-241.

12. T. Onodera, Linearly compact modules and cogenerators, J. Fac. Sci. Hokkaido Univ. Ser. I, 22 (1972), 116-125.

13. F. L. Sandomierski, Linearly compact modules and local Morita duality, in Ring theory (ed. by R. Gordon), 333-346, Academic Press, New York, 1972.

14. D. Zelinsky, Linearly compact modules and rings, Amer. J. Math. 75 (1953), 79-90.

15. H. Zöschinger, Komplementierte Moduln über Dedekindringen, J. Algebra 29 (1974), 42-56.

16. H. Zöschinger, Komplemente als direkte Summanden, Arch. Math. (Basel) 25 (1974), 241-253.

\section{MATHEMATISCHES INSTITUT}

DER UNIVERSITATT MUNCHEN,

BUND ESREPUBLIK DEUTSCHLAND 